\title{
One transformer inrush current calculation method based on field-circuit-network coupled co-simulation
}

\author{
Xiaobin LIANG $^{1, A}$, Yanbo SHEN ${ }^{2}$, Wei WEI ${ }^{1}$ \\ ${ }^{1}$ Sichuan Electric Power Research Institute of GSCC, Chengdu, 610072, China \\ ${ }^{2}$ Electrical Engineering and Automation College, Tianjin University, Tianjin, 300000, China \\ aliangxiaobinxju@126.com
}

Keywords: Field-circuit-network coupled; co-simulation; magnetizing inrush current; transformer; time stepping element method

\begin{abstract}
In this paper, one calculation method based on field-circuit-network coupled co-simulation is presented. Compared with the traditional simplified transformer model, this transformer model takes the nonlinear factors into account, and the accuracy of the simulation results will be improved. The transformer is build with the finite element method by using the software of Maxwell, and circuit of transformer is build by using the software of Simplorer, and the network model is build under Matlab/Simulation's environment. At last, this field-circuit-network coupled model is used to calculate the inrush current of transformer.
\end{abstract}

\section{Introduction}

The power transformer is one kind of basic and important equipment in power system, and its running state directly affects the reliability of the power supply. So, it is very necessary to work on the modeling method of power transformer.

The practical power transformer model is widely used in power system simulation. This modeling method of physical meaning is clear, and is easy to implement the requirements of power system dynamic analysis and calculation. However, this model is obtained by simplified processing, and many nonlinear factors are not taken into account [1-2]. So this model can not accurately reflect many nonlinear factors, it will affect the accuracy of the simulation results, and it even causes serious errors of the analysis. So, it has the significance to study the transformer model with nonlinear factors [3-4]. In this paper, the field-circuit-network coupled modeling method is presented. The transformer is build with the finite element method by using the software of Maxwell. In this field transformer model, the nonlinear factors such as the nonlinear hysteresis curve is considered, and circuit of transformer is build by using the software of Simplorer, and the network model is build under Matlab/Simulation's environment.

\section{The construction of Transformer field-circuit-network coupled model}

\section{Time step finite element model of the transformer}

The two-dimensional finite element model of single-phase transformer is shown in Figure 1, in which the establishment of the "field model" is completed. The modeling parameters of single-phase transformer are shown in table 1. 


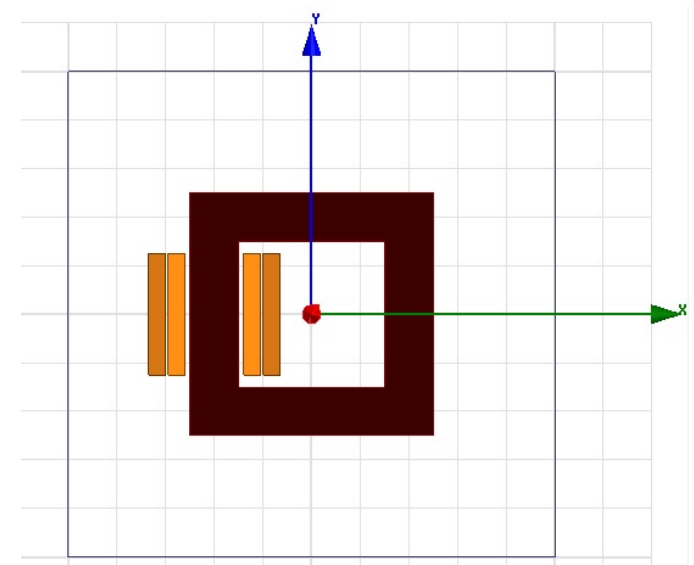

Fig.1 The 2D finite element model of transformer

Table 1. Model parameters of transformer

\begin{tabular}{c|c|c|c}
\hline Parameter & Value & Parameter & Value \\
\hline Voltage class & $35 \mathrm{kV}$ & Rated capacity & $1000 \mathrm{kVA}$ \\
\hline Transformation ratio & 3.33 & Height of coil & $500 \mathrm{~mm}$ \\
\hline Inside length of iron core & $1000 \mathrm{~mm}$ & Length of coil & $70 \mathrm{~mm}$ \\
\hline Outside length of iron core & $600 \mathrm{~mm}$ & Size of solve field & $2000 \mathrm{~mm}$ \\
\hline
\end{tabular}

\section{The establishment of the transformer excitation model}

For the preliminary model, the single-phase transformer excitation source is established in Matlab/Simulink, and only a voltage source is added. So, the establishment of the "network" is completed. The model is shown in figure 2 .

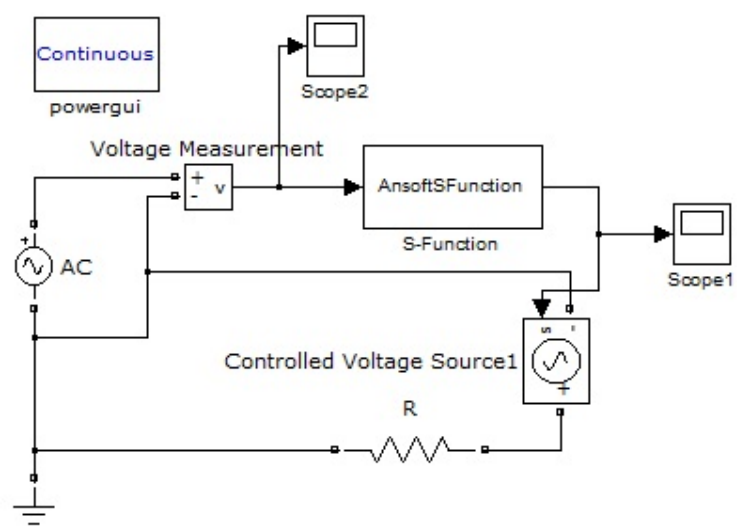

Fig.2 The excitation model of transformer

\section{The connection between the coupled models}

After the completion of the above "field" and "network" models, it needs to establish the connection relationship between the two models, which requires the establishment of the "circuit". The circuit model is finished by Ansoft/Simplorer software, and the established "circuit" model is shown in figure 3. 


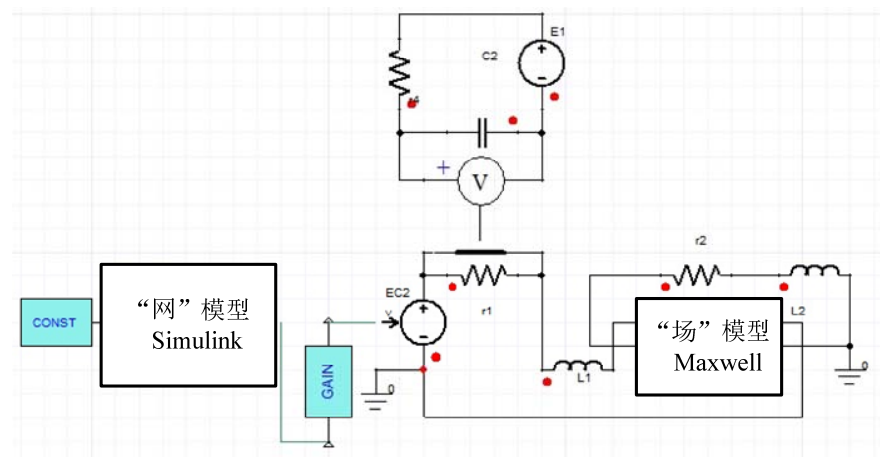

(a) Circuit connection between field model and network model

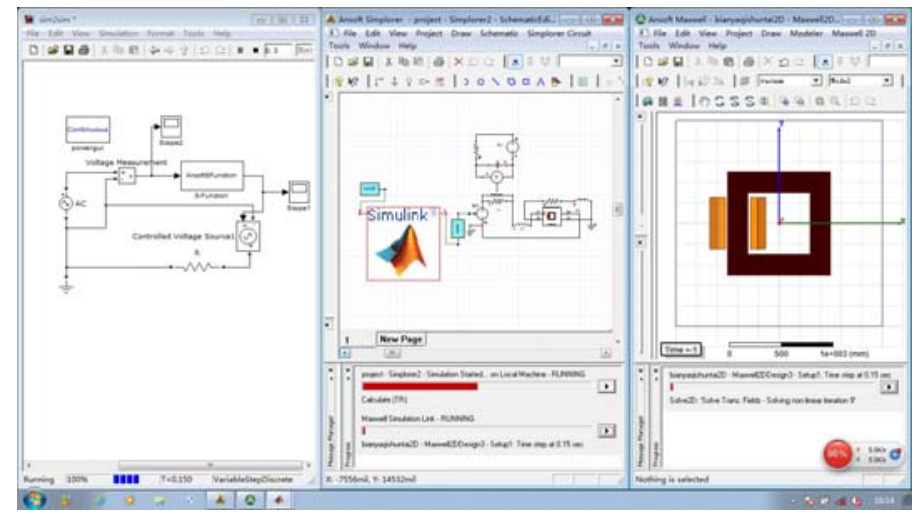

(b) The operation interface of co-simulation

Fig.3 The field-circuit-network coupled model of transformer

\section{Simulation result of the transformer field-circuit-network coupled stepping finite element model}

The simulation result of transformer inrush current

The high voltage side of transformer is added with load voltage, and it can cause excitation inrush current. The simulation result of coupling model is shown in figure 4.

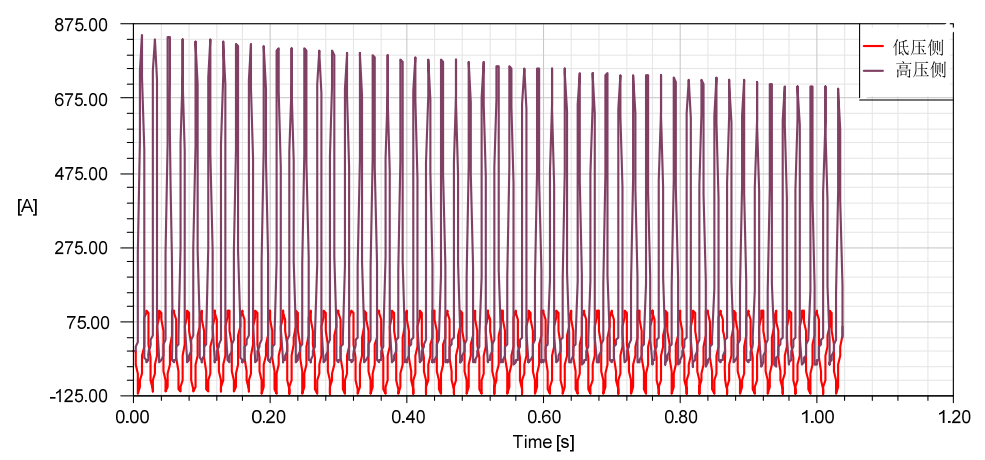

Fig.4 The simulation results of transform's inrush current by two different models

\section{Transformer inrush current Suppression}

In order to eliminate this part of the energy as soon as possible and make high-voltage side current settle down as soon as possible, one $100 \mathrm{ohm}$ resistance is connected to the high side circuit, and it will be removed in 0.2 seconds. The current waveform is shown in figure 5 . It can be found that, the inrush current is suppressed obviously. 


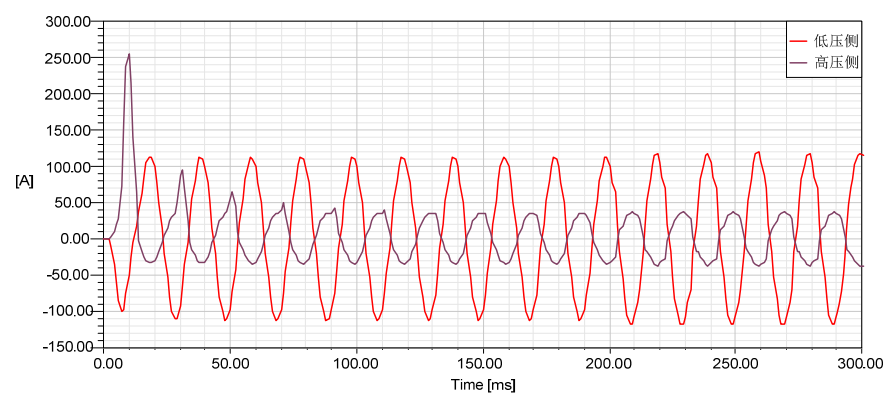

Fig.5 The current of transformer when closing resistor is connected in short-time

The induced voltage waveform is presented in Figure 6, and the flux in transformer's iron core is presented in Figure 7.

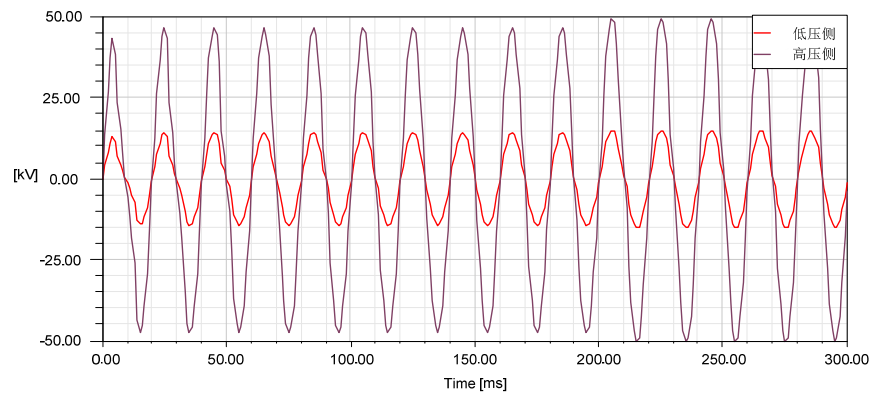

Fig.6 The induced voltage of transformer

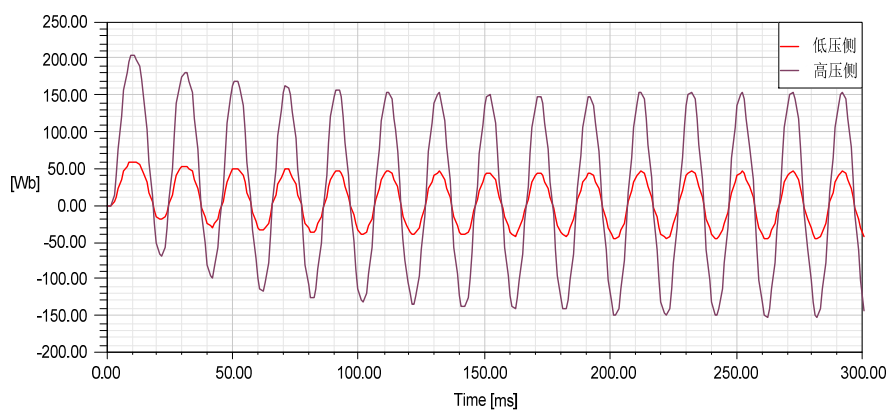

Fig.7 The flux-linkage of transformer

At this time, after the transformer is stable, the magnetic distribution in the iron core is shown in figure 8. Obviously, the maximum flux density in core don't passed knee point of B-H curve (about 1.6 T), which means that the transformer operates in unsaturated state.

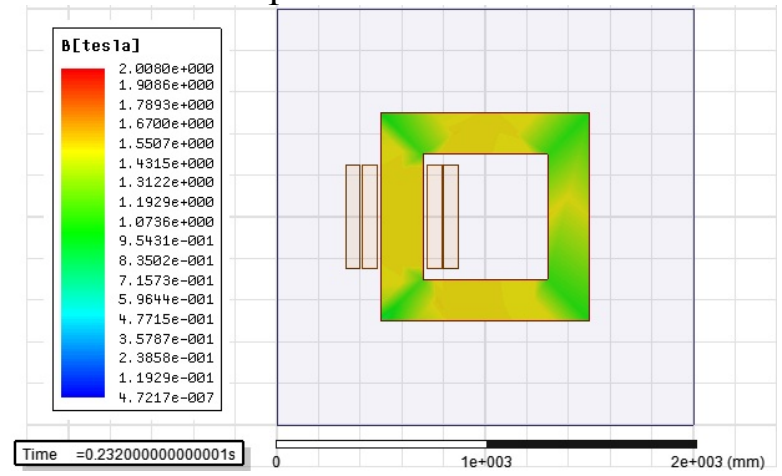

Fig.8 The flux density in the iron core of transformer

\section{Conclusion}

This paper introduced one field-circuit-network coupled model of transformer. The transformer is build with the finite element method by using the software of Maxwell. In this field transformer model, the nonlinear factors such as the nonlinear hysteresis curve is considered, and circuit of transformer is build by using the software of Simplorer, and the network model is build under Matlab/Simulation's environment. 
The transformer field-circuit-network coupled time step model can give play to the advantages of different software, and it provides a very meaningful tool for the transformer simulation analysis.

\section{References}

[1] Leon F D, Semlyen A. Complete transformer model for electromagnetic transients[J]. IEEE Trans on Power Delivery, 1994, 9(1): 231-239.

[2] Wang Xue, Wang Zengping. Study of simulation of transformer with internal fault[J]. Power System Technology, 2004, 28(12): 50-52.

[3] Wang Xi-ping, Li Wen-cai, Li yan, Lu wen-mei. The building and emulation of distribution transformer model based on simulink[J]. Techniques of antomation and application, 2015, 09: 124-132.

[4] Yang Lishui, Yang $\mathrm{xu}, \mathrm{Xu}$ yan. A nonlinear model to calculate post-internal fault parameters of power transformer[J]. Power system technology, 2009:20-0183-06. 